Losada-Puente, L.; Muñoz-Cantero, J.M. y Espiñeira-Bellón, E.M. (2018). Adaptación del Cuestionario de Evaluación de la Calidad de Vida de Alumnos adolescentes (CCVA, Gómez-Vela \& Verdugo, 2009) en Galicia (España). Revista de Investigación Educativa, 36(2), 331-347.

DOI: http://dx.doi.org/10.6018/rie.36.2.320171

\title{
Adaptación del Cuestionario de Evaluación de la Calidad de Vida de Alumnos adolescentes (CCVA, Gómez-Vela \& Verdugo, 2009) en Galicia (España)
}

\author{
Adaptation of the Questionnaire of Quality of Life \\ Assessment for teenagers (CCVA, Gómez-Vela \& Verdugo, 2009) \\ in Galicia (Spain)
}

\author{
Luisa Losada-Puente, Jesús Miguel Muñoz-Cantero y Eva María Espiñeira-Bellón \\ Departamento de Didácticas Específicas y Métodos de Investigación en Educación. \\ Facultad de Ciencias de la Educación. Universidad de A Coruña (España).
}

\begin{abstract}
Resumen
La producción y difusión de modelos e investigaciones en calidad de vida ha optado por un enfoque multidimensional. Nuestro objetivo es analizar el funcionamiento del Cuestionario de Evaluación de la Calidad de Vida de Alumnos adolescentes (CCVA, Gómez-Vela $\mathcal{E}$ Verdugo, 2009) que evalúa siete dimensiones del Modelo de Calidad de Vida en la adolescencia, diseñado por los autores. Se aplica a una muestra de 2220 estudiantes (12-19 años). Se realiza un Análisis Factorial Confirmatorio (AFC) y un análisis correlacional entre variables latentes. Se comprueba el poder discriminativo de los ítems (IHc>.15; ID>.4) y la fiabilidad ( $\alpha=0.914$; rango $\alpha=0.746$ y 0.928). El AFC compara el ajuste del modelo original de Gómez-Vela y Verdugo (2004, 2006). La ausencia de ajuste lleva a proponer un modelo alternativo. Dicho modelo introduce la dimensión derechos, modifica el concepto integración por el de inclusión, y unifica las dimensiones bienestar emocional y físico. Dicho modelo
\end{abstract}

Correspondencia: Luisa Losada Puente, email luisa.losada@udc.es, Facultad de Ciencias de la Educación, campus de Elviña, s/n, 15071 A Coruña (España). 
presenta un buen ajuste, con evidencias de fiabilidad (IFC=0.936) y validez $(A V E=.640)$, presentando correlaciones elevadas entre autodeterminación y derechos, y bajas en el resto de casos. La evaluación de la calidad de vida es clave para analizar las necesidades de la juventud y planificar intervenciones individualizadas. Pero las diferencias entre regiones, patrones culturales y formas de vida local exigen atender a las características propias de cada grupo de evaluación.

Palabras clave: bienestar del estudiante; satisfacción con la vida; adolescencia; análisis factorial.

\begin{abstract}
The production and dissemination of models and researches on quality of life has focused on a multidimensional approach. Our aim is to analyze the functioning of the Questionnaire of Assessment of the Quality of Life of Adolescent students (CCVA, Gómez-Vela EVerdugo, 2009) that assess seven dimensions of the Model of Quality of Life in the adolescence, designed by the authors. A sample of 2220 students (aged 12-19) is used. A Confirmatory Factor Analysis (CFA) and a correlational analysis between latent variables are carried out. The discriminant power of the items $(H I c>.15 ;$ ID $>.4)$ and the reliability $(\alpha=.914$; rank $\alpha=0.746$ y 0.928$)$ are tested. The CFA check the fit of the original Gómez-Vela and Verdugo $(2004,2006)$ model. The absence of adjustment leads to propose an alternative model. This model introduces the dimension rights, modifies the concept of integration by inclusion one, and unifies the emotional and physical well-being dimensions. This one presents the best fit to the data, with evidences of reliability $(C R I=.936)$ and validity $(E V A=.640)$. High correlation between self-determination and rights is shown. The assessment of quality of life is a key to analyze the needs and demands of teenagers and to plan interventions from an individual approach. However, the differences between regions, cultural patterns, and local ways of life require attention to the characteristics of each evaluation group.
\end{abstract}

Keywords: student welfare; life satisfaction; youth; factor analysis.

\title{
Introducción
}

La calidad de vida es un constructo de amplia trascendencia en todos los contextos de la actividad humana y a lo largo del ciclo vital. El interés científico por este constructo se expande a diversos campos del conocimiento, destacando notablemente en las Ciencias Sociales, cuyo objeto principal de estudio es la percepción de cada individuo acerca de su vida, lo que siente y piensa sobre ella, su valoración de los bienes y servicios de los que dispone y que utiliza, de las relaciones e interacciones con otras personas, y de las situaciones que experimenta en cada contexto concreto (Claes, van Hove, van Loon, Vandevelde, \& Schalock, 2010; Vega, Jenaro, Flores, Cruz, \& Lerdo, 2013; Verdugo, Sainz, Gómez-Sánchez, \& Gómez-Santamaría, 2011).

Este enfoque amplio y subjetivo, que aglutina aspectos como el bienestar, la felicidad, la satisfacción, la salud, etc., dificulta ofrecer la definición de este constructo. Pero más allá de tratar de ofrecer una definición única, los esfuerzos se han centrado en identificar los predictores clave de una vida de calidad para el individuo. 
En el año 2000, the Special Interest Research Group on Quality of Life de la Asociación Internacional para el Estudio Científico de las Discapacidades Intelectuales (International Association for the Scientific Study of Intellectual Disabilities, IASSSID SIRG, 2000) señala varios principios generales para la conceptualización, medición y aplicación de la calidad de vida. Fruto de ello, comienzan a desarrollarse algunos modelos que pretenden operativizarla a través de dimensiones que caracterizan la vida del individuo. Pese a las discrepancias de dichos modelos en lo que respecta a sus dimensiones y su importancia, proponen algunas nociones esenciales (GómezVela \& Verdugo, 2006; Verdugo et al., 2011):

1. El individuo es el agente causal de todo el proceso; quien valora su percepción promueve el cambio en los servicios y actividades en que participa.

2. La calidad de vida es un constructo multidimensional y debe ser abordado desde una perspectiva holística.

3. La calidad de vida es un concepto de igual importancia para todos los individuos y, por tanto, sus dimensiones, su evaluación y aplicación serán también las mismas.

En base a estos principios, se han desarrollado diversas líneas de investigación con enfoques de estudio variados: (a) calidad de vida individual (Gómez-Sánchez, Peña, Arias, \& Verdugo, 2016; Morisse, Vandemaele, Claes, Claes, \&Vandevelde, 2013; Schalock, Verdugo, Gómez, \& Reinders, 2016), sobre todo, en la adultez (Balboni, Coscarelli, Giunti, \& Schalock, 2013; Vega et al., 2013) y en la vejez (Beadle-Brown et al., 2015; Escarabajal, Martínez, \& Salmerón, 2015); (b) calidad de vida familiar (Arellano \& Peralta, 2013, 2015), y (c) calidad de vida relacionada con la salud (Biggs \& Carter, 2016).

No obstante, la producción y difusión de modelos e investigaciones en calidad de vida en la adolescencia ha sido, hasta hace relativamente poco tiempo, mucho menor. Ello puede deberse, en primer lugar, a la idea tradicional de la educación como tiempo de preparación para la vida adulta y no como fase importante en sí misma; en segundo lugar, al uso de modelos e instrumentos propios de la etapa adulta; y finalmente, a la complejidad que representa en sí misma esta etapa del desarrollo, pues en ella confluyen múltiples factores biológicos, psíquicos, sociales y contextuales que interactúan como facilitadores o barreras a la mejora del bienestar de cada joven (Alfaro, Casas, \& López, 2015; Gómez-Vela, 2007; Gómez-Vela, Verdugo, \& González-Gil, 2007; Higuita \& Cardona, 2015; Muntaner, 2013).

En el terreno de la educación escolar, la calidad de vida se convierte en un concepto clave para analizar la realidad educativa y social del estudiante, a fin de reconducir el proceso educativo hacia la satisfacción de las necesidades, intereses y deseos del alumnado frente al énfasis en los aspectos curriculares y organizativos (Muntaner, 2013). $\mathrm{Al}$ respecto, podemos referirnos a uno de los modelos teóricos más actuales y que es sustento de este estudio: el Modelo de Calidad de Vida en la Adolescencia (Gómez-Vela \& Verdugo, 2004, 2006), que adopta una perspectiva multidimensional para definir el constructo, y sostiene que la importancia que el adolescente ofrece a cada área de su vida podrá variar respecto de otros jóvenes y a lo largo de la vida. 


\section{Método}

\section{Objetivos}

Los objetivos de este estudio son tres: primero, validar el Cuestionario de Evaluación de la Calidad de Vida de Alumnos adolescentes (CCVA, Gómez-Vela \& Verdugo, 2009; con su autorización) en Galicia; segundo, corroborar la adecuación del Modelo de Calidad de Vida de la adolescencia de Gómez-Vela \& Verdugo $(2004,2006)$; y tercero, analizar las relaciones existentes entre las dimensiones que componen el constructo de calidad de vida.

\section{Población y Muestra}

La población del estudio es el alumnado que cursa Educación Secundaria Obligatoria (ESO) o Bachillerato en la Comunidad Autónoma de Galicia, formada por un total de 121977 adolescentes entre 12 y 19 años en el año 2015 (Instituto Galego de Estadística, IGE, 2015).

Para la selección de la muestra, el personal investigador contactó con los centros educativos de la Comunidad Autónoma de Galicia para explicar el estudio y obtener su permiso para aplicar el cuestionario. El CCVA fue aplicado durante el curso 2014/2015 en aquellos centros donde se obtuvo consentimiento del equipo directivo y las familias. De tal forma, la muestra quedó compuesta por 2220 estudiantes escolarizados en Centros Educativos Públicos (76.17\%), Concertados (16.62\%) y Privados (7.21\%), de las cuatro provincias gallegas: A Coruña (22.68\%), Lugo (11.34\%), Orense $(9.27 \%)$ y Pontevedra $(17.53 \%)^{1}$, de entornos urbanos y rurales. La edad media de los participantes es 14.29 (D.T.= 1.81), siendo 1230 hombres (55.41\%) y 990 mujeres $(44.59 \%)$.

\section{Instrumento}

El CCVA es un instrumento diseñado para su aplicación a adolescentes de 12 a 18 años. Presenta una estructura formada por 56 ítems (escala Likert 1-4) que evalúa las siete dimensiones de la calidad de vida (bienestar emocional, bienestar material, bienestar físico, relaciones interpersonales, integración/presencia en la comunidad, desarrollo personal y autodeterminación), así como 10 ítems destinados a controlar la deseabilidad social y siete pares de ítems que controlan la aquiescencia. El uso de este instrumento en España aún no es muy extendido, si bien su aplicación inicial mostró una adecuada consistencia en la escala total $(\alpha=.84)$ y en sus dimensiones (rango $\alpha=0.58-\alpha=0.82$ ) y adecuada validez de contenido y de constructo (Gómez-Vela \& Verdugo, 2009).

1 Los porcentajes hacen referencia al valor total de cada provincia en relación a su totalidad. 


\section{Procedimiento de análisis de datos}

Al finalizar la recogida de datos, se controló la presencia de aquiescencia (ofrecer la misma respuesta a más de tres pares de ítems inversos), deseabilidad social (atribuirse cualidades personales socialmente deseables, obteniendo una puntuación de 30 o superior sobre 40 en las preguntas destinadas a tal fin) y la omisión de respuestas en varios ítems. Fueron eliminados 176 cuestionarios que presentaban una o varias de estas situaciones, quedando la muestra final compuesta por 2220 estudiantes.

Los ítems destinados al control de la aquiescencia y la deseabilidad social fueron excluidos de los posteriores análisis. Cabe también destacar que este instrumento presenta 6 ítems inversos, cuyas respuestas han sido invertidas antes de efectuar los análisis. Los análisis estadísticos, apoyados por el paquete estadístico IBM SPSS 23, se basaron en el análisis correlacional de los ítems, a través del cálculo del Índice de Homogeneidad Corregida (IHc) y del Índice de Discriminación de los ítems (ID), así como otros cálculos relacionados con la preparación de los datos para el desarrollo de un Análisis Factorial Confirmatorio (AFC). Dicho análisis se llevó a cabo mediante el programa IBM SPSS AMOS 23, en base a los coeficientes de correlación entre las variables latentes del modelo más eficiente para explicar el constructo de calidad de vida.

\section{Resultados}

\section{Análisis correlacional de los ítems}

En primer lugar, se analiza la correlación entre cada ítem del CCVA y la puntuación total comprobando el poder discriminador de cada ítem al test, eliminando la contribución de éste último al ítem mediante el Índice de Homogeneidad Corregido (IHc). También se estudia el Índice de Discriminación (ID).

Se han encontrado las puntuaciones más bajas en los ítems 26A y 26B, con valores inferiores a 2.3. La media más elevada está en ítems pertenecientes a la dimensión relaciones interpersonales $(1 \mathrm{~A}$ y $17 \mathrm{~A} / \mathrm{B} / \mathrm{C}$ ) y bienestar físico (5 y 15), con valores superiores a 3.5. En cuanto al IHc, los valores de los ítems superan el 0.15 en todos los casos (siendo valores $>0.5$ en los ítems $4,38,48$ y 56), por lo que no es necesaria la exclusión de ninguno de ellos, por ser el IHc positivo (Mateo \& Martínez, 2008). Generalmente, los ítems (trasformando los valores a una escala 0-1, según sea valoración baja o alta) presentan un elevado poder discriminatorio pues, al calcular el índice de discriminación $[\mathrm{ID}=(\mathrm{AS}-\mathrm{AI}) /(\mathrm{N} / 2)]$, se obtienen valores entre $\mathrm{ID}=0.35 \mathrm{e} \mathrm{ID}=0.80$.

\section{Análisis Factorial Confirmatorio}

Dada la existencia de un modelo teórico subyacente a la construcción de la escala y teniendo evidencias del poder discriminatorio de sus ítems, se realiza un Análisis Factorial Confirmatorio (AFC) a fin de corroborar la idoneidad de los indicadores para evaluar las variables latentes. Previamente, se decidió la parcelación de items o "promedio de dos o más ítems y el uso de los resultados de la suma o media como la unidad básica del análisis en el Modelo de Ecuaciones Estructurales" (Bandaloos \& Finney, 2009, p. 269). 
Nos basamos en el Modelo de Calidad de Vida en la adolescencia (Gómez-Vela \& Verdugo, 2004, 2006) y en los indicadores propuestos por los autores para definir cada una de las dimensiones (Tabla 1).

La estructura original del CCVA se presenta en la tabla 1, donde cada dimensión se compone de dos o tres indicadores evaluados por un conjunto de ítems agrupados en base a su identidad conceptual y a la presencia de correlación positiva significativa $(\mathrm{p}<.000)$. La fiabilidad de los indicadores en cada dimensión es satisfactoria, oscilando entre .746 y .928 .

Tabla 1.

Estructura original del CCVA: dimensiones, indicadores, ítems y su consistencia interna

\begin{tabular}{|c|c|c|c|}
\hline Dimensiones & Indicadores & Ítems & $\alpha$ \\
\hline \multirow{2}{*}{$\begin{array}{l}\text { Relaciones } \\
\text { interpersonales } \\
\text { (RRII) }\end{array}$} & Relaciones valiosas con la familia & $1 \mathrm{~A}, 1 \mathrm{~B}, 17 \mathrm{~A}, 17 \mathrm{~B}, 17 \mathrm{C}$ & \multirow[b]{2}{*}{.807} \\
\hline & $\begin{array}{l}\text { Redes de apoyo social de amigos, } \\
\text { compañeros y conocidos }\end{array}$ & $9,24,31,38,53$ & \\
\hline \multirow{2}{*}{$\begin{array}{l}\text { Bienestar material } \\
\text { (BMA) }\end{array}$} & Estatus socioeconómico familiar & $19,47,55$ & \multirow{2}{*}{.861} \\
\hline & Pertenencias, alimentación y vivienda & $3,26 \mathrm{~A}, 26 \mathrm{~B}, 39$ & \\
\hline \multirow{2}{*}{$\begin{array}{l}\text { Desarrollo personal } \\
(\mathrm{DEP})\end{array}$} & $\begin{array}{l}\text { Habilidades, capacidades y } \\
\text { competencias }\end{array}$ & $6,14,42$ & \multirow{2}{*}{.796} \\
\hline & $\begin{array}{l}\text { Educación, actividades significativas y } \\
\text { oportunidades formativas }\end{array}$ & $35,50,57 \mathrm{~A}, 57 \mathrm{~B}$ & \\
\hline \multirow{2}{*}{$\begin{array}{l}\text { Bienestar emocional } \\
(\mathrm{BEM})\end{array}$} & Satisfacción, felicidad, bienestar general & $4,27,48,56$ & \multirow[b]{2}{*}{.837} \\
\hline & $\begin{array}{l}\text { Seguridad personal/emocional, } \\
\text { autoconcepto, autoestima y autoimagen }\end{array}$ & $12,33,40$ & \\
\hline \multirow{2}{*}{$\begin{array}{l}\text { Bienestar físico } \\
\text { (BEF) }\end{array}$} & Salud y estado físico & $5,13,41$ & \multirow[t]{2}{*}{.746} \\
\hline & Movilidad & 15,28 & \\
\hline \multirow{3}{*}{$\begin{array}{l}\text { Integración/ } \\
\text { Presencia en } \\
\text { comunidad (INT) }\end{array}$} & Presencia de servicios en la comunidad & $2,32 \mathrm{~A}, 32 \mathrm{~B}, 32 \mathrm{C}$ & \multirow{3}{*}{.827} \\
\hline & $\begin{array}{l}\text { Información y participación en los } \\
\text { servicios de la comunidad }\end{array}$ & $18 \mathrm{~A}, 18 \mathrm{~B}, 18 \mathrm{C}, 46$ & \\
\hline & $\begin{array}{l}\text { Integración y normalización, estatus } \\
\text { dentro del grupo social }\end{array}$ & $25,54 \mathrm{~A}, 54 \mathrm{~B}, 54 \mathrm{C}$ & \\
\hline \multirow{2}{*}{$\begin{array}{l}\text { Autodeterminación } \\
\text { (AUT) }\end{array}$} & $\begin{array}{l}\text { Autonomía, toma de decisiones y } \\
\text { elecciones personales }\end{array}$ & $7,36,22,29$ & \multirow[t]{2}{*}{.928} \\
\hline & Control personal y capacitación & $43,51,58$ & \\
\hline
\end{tabular}


Teniendo en cuenta estos valores, resulta procedente preparar los datos para realizar el AFC, atendiendo a aspectos como (Arias, 2008; Kline, 2015; Malone \& Lubansky, 2014):

- El nivel de medida: empleamos el Método de Distribución Libre Asintótica (Asymptotical Distribution Free, ADF), que nos permite analizar variables ordinales que no se ajustan a los criterios de la normalidad.

- Valores por indicador: se emplean las puntuaciones típicas (zi).

- Normalidad univariante y multivariante: mediante los contrastes de asimetría y curtosis, se rechaza la hipótesis de simetría $[z(\mathrm{G} 1)> \pm 1.96]$ y de distribución mesocúrtica $[z(\mathrm{G} 2)> \pm 1.96]$. Así mismo, el contraste conjunto entre asimetría y curtosis indica el incumplimiento del supuesto de normalidad, pues en todos los casos $k^{2}> \pm 5.99$. La prueba de normalidad de Kolmogorov-Smirnov, con corrección de significación de Lilliefors indica el rechazo a la hipótesis nula de normalidad de las variables $(\mathrm{p}<.05)$. En lo que respecta a los valores anómalos, se calcula el estadístico Distancia de Mahalanobis $\left(\mathrm{D}^{2}\right)$, que se distribuye aproximadamente como $\chi 2$ con tantos grados de libertad como número de variables existan (Arias, 2008). Hallamos 54 casos anómalos ( $>$ >.001), que se pueden considerar errores concernientes a la idiosincrasia de la muestra, formada por grupos heterogéneos de alumnado.

- Datos perdidos: El porcentaje de los casos perdidos oscila entre 1.8 y el $3.6 \%$ en los ítems P1B, P17A y P17B, no siendo lo suficientemente grandes como para eliminarlos. Se trata de valores perdidos por las características del sujeto ante cuestiones como "mantengo buenas relaciones con mis abuelos", "mantengo buenas relaciones con mis tíos" o "mantengo buenas relaciones con mis primos", puesto que algunos sujetos no cuentan con dichos familiares. Se emplea el método de imputación múltiple para su tratamiento (Lang \& Little, 2016).

- Número mínimo de observaciones: este estudio supera las recomendaciones, en lo que respecta al empleo de muestras grandes (Arias, 2008; Kline, 2015).

- Número de indicadores total y por variable latente: el procedimiento de item parceling (parcelación de ítems, presentados en la tabla 1) permite subsanar el riesgo de sobreestimación de $\chi^{2}$. La matriz se compone de 15 indicadores (parcelas), dos para cada variable latente, excepto Integración/Presencia en la comunidad que incluye tres.

Una vez preparados los datos, se siguió un proceso en varios pasos (Kelloway, 2014; Kline, 2015): especificación del modelo, identificación, estimación de parámetros, evaluación de ajuste, reespecificación e interpretación de los resultados. En primer lugar, se especifica el Modelo de Calidad de Vida en la Adolescencia (Gómez-Vela \& Verdugo, 2004, 2006) compuesto por siete dimensiones correlacionadas, que son adoptadas como variables exógenas para definirlo: Bienestar emocional (F1), Integración/ Presencia en la comunidad (F2), Relaciones interpersonales (F3), Desarrollo personal (F4), Bienestar físico (F5), Autodeterminación (F6) y Bienestar material (F7). 
La identificación se basa en la regla de los grados de libertad (Kenny \& Milan, 2014) que incluye un total de 22 varianzas y 21 covarianzas y cuenta con 51 parámetros a estimar y 69 grados de libertad (gl), de modo que está sobreidentificado.

Seguidamente, se estimaron los parámetros a partir de las varianzas y covarianzas muestrales mediante el método de Distribución Libre Asintótica (ADF). La representación gráfica del resultado se presenta en la Figura 1.

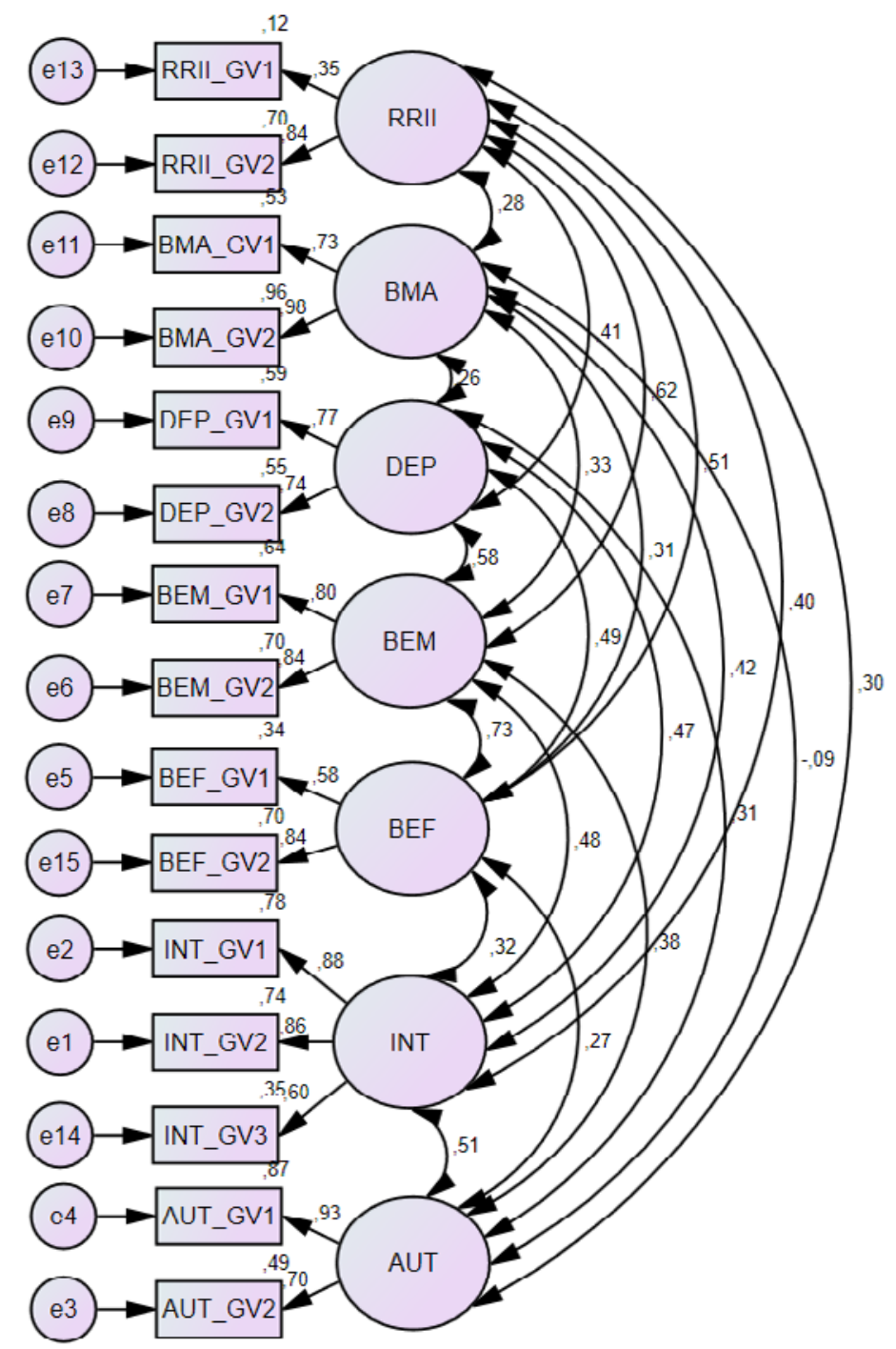

Figura 1. Parámetros estandarizados del Modelo 1.

Calidad de Vida (Gómez-Vela y Verdugo, 2004, 2006). 
Tras estimar los parámetros, se evaluó la adecuación del modelo teórico a los datos, analizando el grado de ajuste global con el que se comprobó en qué medida el modelo hipotetizado reproducía las relaciones existentes en la matriz de correlaciones.

La figura 1 muestra los coeficientes de correlación estandarizados entre factores, y entre éstos y las variables. Las cargas factoriales en cada variable muestran, generalmente, valores adecuados (superiores a .7 excepto en RRII_GV1, BEF_GV1 e INT_GV3), con errores de predicción que oscilan entre .12 (RRII_GV1) y .96 (BMA_GV2).Todos los ítems fueron estadísticamente significativos $(\mathrm{p}<.01)$.

Se contrastaron varios índices de ajuste pues, en su mayoría, no ofrecen por si solos toda la información requerida para valorar el modelo (Kelloway, 2014; Kline, 2015). Como se presenta en la Tabla 3, se optó por la combinación que reúne los índices $\chi^{2}$, la razón $\chi^{2}$ sobre los grados de libertad (CMIN/gl), ajuste comparativo (CFI), bondad de ajuste (GFI), su versión ajustada (AGFI) y el error de aproximación de la raíz cuadrada media (RMSEA) (Escobedo Portillo, Hernández Gómez, Estebanéz Ortega, \& Martínez Moreno, 2016).

El valor de $\chi^{2} / \mathrm{gl}$ es de 35.46 ( $\left.\mathrm{p}<.001\right)$, muy superior al valor óptimo que debe ser inferior a 3, lo que indica que el ajuste del modelo no es el adecuado. No obstante, dada su restrictividad, se opta por examinar otros índices que permitan subsanar las limitaciones que presenta el primero. Concretamente, siguiendo las recomendaciones de Arias (2008) y West, Taylor, \& Wu (2015), se evalúa el ajuste parcial absoluto mediante la Raíz del Residuo Cuadrático Promedio (RMR > 1) y el Índice de Bondad de Ajuste (GFI=.901), que resultan adecuados, y su versión ajustada (AGFI=.837), en este caso, deficiente. Así mismo, se emplea el Error de Aproximación Cuadrático Medio (RMSEA), que obtiene valores inferiores a .80 (RMSEA $=.72)$, siendo la probabilidad de que el valor RMSEA sea $<.05$ inferior al $1 \%$.

Así, tomando los datos obtenidos de la estimación de los parámetros del modelo y del cálculo de los estadísticos de bondad de ajuste, se puede señalar que, pese a que este modelo está teóricamente identificado y validado mediante AFE, su identificación empírica mediante el AFC presenta problemas en su contraste en población gallega.

Por tal motivo, reespecificamos el modelo, ajustándolo y adaptándolo a la realidad que es objeto de estudio, y tomando como base las teorías previas que definen y operativizan el constructo (Gómez-Vela \& Verdugo, 2004, 2006; Schalock \& Verdugo, 2002).

\section{Reespecificación del modelo de calidad de vida}

La reespecificación del modelo propuesto por Gómez-Vela \& Verdugo (2004, 2006) atiende a la intención de introducir modificaciones acordes con el Modelo Heurístico (Schalock \& Verdugo, 2002) diseñado para la población adulta, y en el que se tienen en cuenta las dimensiones inclusión y derechos. De tal manera, se proponen las siguientes siete dimensiones: Autodeterminación (F1), Bienestar emocional y físico (F2), Inclusión (F3), Relaciones interpersonales (F4), Bienestar Material (F5), Desarrollo Personal (F6) y Derechos (F7), que integran dos indicadores cada uno, excepto bienestar emocional y físico que recoge tres, al estar compuesta por 11 ítems (tabla 2; figura 2). 
Tabla 2.

Estructura modificada del CCVA: dimensiones, indicadores, ítems y su consistencia interna

\begin{tabular}{|c|c|c|c|}
\hline Dimensiones & Indicadores & Ítems & $\alpha$ \\
\hline \multirow{2}{*}{$\begin{array}{l}\text { Relaciones } \\
\text { interpersonales } \\
\text { (RRII) }\end{array}$} & Relaciones valiosas con la familia & $\begin{array}{l}1 \mathrm{~A}, 1 \mathrm{~B}, 17 \mathrm{~A}, 17 \mathrm{~B} \\
17 \mathrm{C}\end{array}$ & .709 \\
\hline & $\begin{array}{l}\text { Redes de apoyo social de amigos, } \\
\text { compañeros y conocidos }\end{array}$ & $9,24,31,38,53$ & .861 \\
\hline \multirow[t]{2}{*}{$\begin{array}{l}\text { Bienestar material } \\
\text { (BMA) }\end{array}$} & Estatus socioeconómico familiar & $19,47,55$ & .881 \\
\hline & Pertenencias, alimentación y vivienda & $3,26 \mathrm{~A}, 26 \mathrm{~B}, 39$ & .663 \\
\hline \multirow{2}{*}{$\begin{array}{l}\text { Desarrollo personal } \\
\text { (DEP) }\end{array}$} & Habilidades, capacidades y competencias & $6,14,42$ & .728 \\
\hline & $\begin{array}{l}\text { Educación, actividades significativas y } \\
\text { oportunidades formativas }\end{array}$ & $35,50,57 \mathrm{~A}, 57 \mathrm{~B}$ & 686 \\
\hline \multirow{3}{*}{$\begin{array}{l}\text { Bienestar emocional } \\
\text { y físico (BEF) }\end{array}$} & Satisfacción, felicidad, bienestar general & $4,27,48,56$ & .907 \\
\hline & $\begin{array}{l}\text { Seguridad personal/emocional, } \\
\text { autoconcepto, autoestima y autoimagen }\end{array}$ & $28,33,40$ & .694 \\
\hline & Salud, movilidad y estado físico & $5,13,15,41$ & 692 \\
\hline \multirow[b]{2}{*}{ Inclusión (INC) } & Presencia de servicios en la comunidad & $2,32 \mathrm{~A}, 32 \mathrm{~B}, 32 \mathrm{C}$ & .773 \\
\hline & $\begin{array}{l}\text { Información y participación en los } \\
\text { servicios de la comunidad }\end{array}$ & $18 \mathrm{~A}, 18 \mathrm{~B}, 18 \mathrm{C}, 46$ & .709 \\
\hline \multirow{2}{*}{ Derechos (DER) } & Integración y normalización & $54 \mathrm{~A}, 54 \mathrm{~B}, 54 \mathrm{C}$ & .909 \\
\hline & Estatus dentro del grupo social & $12,25,36$ & .921 \\
\hline \multirow{2}{*}{$\begin{array}{l}\text { Autodeterminación } \\
\text { (AUT) }\end{array}$} & $\begin{array}{l}\text { Autonomía, toma de decisiones y } \\
\text { elecciones personales }\end{array}$ & $7,22,29$ & .889 \\
\hline & Control personal y capacitación & $43,51,58$ & .825 \\
\hline
\end{tabular}

Como se observa en la tabla 2, cada dimensión se compone de dos o tres indicadores evaluados por un conjunto de ítems agrupados en base a su identidad conceptual y a la presencia de correlación positiva significativa $(\mathrm{p}<.000)$. La fiabilidad de los indicadores es elevada, oscilando entre .663 y .921.

Los resultados, que se muestran en la Tabla 3, presentan la comparativa entre el Modelo 1 de Gómez-Vela y Verdugo, anteriormente analizado, y el Modelo 2 propuesto para este estudio, junto con su reformulación que incluye errores correlacionados; a saber: en primer lugar, en el factor Bienestar Emocional y Físico (e8-e15 y e7-e15); en 
segundo lugar, entre Bienestar Material y Desarrollo Personal (e4-e5) y, finalmente, entre Bienestar Emocional y Físico e Inclusión (e8-e9). Como se indicó, el modelo 1 presenta valores deficientes que indican una falta de ajuste y llevan a proponer la existencia de medidas diferenciadas para cada factor. La bondad de ajuste es mejor, como se puede comprobar en la tabla 3 y en la figura 2, para el Modelo 4 de siete factores con errores correlacionados (EC2) en el que la relación entre $\chi 2$ y grados de libertad es 3.02. El CFI supera holgadamente el nivel de .900, un valor del cual se puede considerar un ajuste aceptable. El GFI estaba por debajo del nivel de .900 en el Modelo 1, pero lo excede en los demás casos. El valor de RMR se mantiene por debajo de .050, lo que también indica un mejor ajuste en el modelo final. Finalmente, un RMSEA por debajo de .080 es considerado aceptable, y los más cercanos a .050 se consideran óptimos. De acuerdo con estos índices, el modelo 4 de siete factores con EC2 proporciona el mejor ajuste.

Tabla 3.

Estadísticos de bondad de ajuste de los modelos de calidad de vida

\begin{tabular}{lcccccc}
\hline & $\chi^{2} / g l$ & RMR & GFI & AGFI & RMSEA & $P$ \\
\hline Modelo 1 & 35.460 & .061 & .887 & .803 & $.125[.068-.076]$ & .000 \\
Modelo 2 & 9.460 & .042 & .940 & .893 & $.062[.057-.066]$ & .000 \\
Modelo 3 EC1 & 3.445 & .026 & .979 & .961 & $.033[.028-.038]$ & .000 \\
Modelo 4 EC2 & 3.020 & .025 & .983 & .966 & $.030[.025-.035]$ & .000 \\
\hline
\end{tabular}

Nota. $\chi^{2}$ : Chi-Cuadrado; gl. Grados de libertad; RMR: Raíz del Residuo Cuadrático Promedio; GFI: Índice de Bondad de Ajuste; AGFI: Índice de Bondad de Ajuste Corregido; RMSEA: Error de Aproximación Cuadrático Medio.

A continuación, se estudiaron las correlaciones estandarizadas entre variables y factores (figura 2) y entre factores (tabla 4) para confirmar, por un lado, la validez de constructo del instrumento y, por otro lado, su validez discriminante, atendiendo así a la correlación entre las variables latentes, atenuadas por el error de medición (+/- 2 veces el error de medición).

Las cargas factoriales sobre las variables observadas que se presentan en la figura 2 muestran valores adecuados, siendo en casi todos los casos superiores a .70 (excepto en RRII_1, que aglutina ítems referidos a las relaciones con los padres/madres, hermanos/as y familiares próximos). La carga más elevada se sitúa en la parcela DER_2 con ítems acerca del estatus del estudiante dentro del grupo social.

Respecto a los coeficientes de correlación entre factores del modelo 4 (ver Tabla 4) alcanzan valores promedio, que oscilan entre .204 (entre relaciones interpersonales y derechos) y .885 (entre autodeterminación y derechos). La ausencia de correlaciones entre factores cercanos a 1 descarta la posibilidad de que dos factores representen la misma dimensión. Esto respalda la validez discriminante del instrumento, que tendría dimensiones suficientemente diferentes. 




Figura 2. Parámetros estandarizados del Modelo 4. Calidad de Vida con EC2.

Tabla 4

Matriz de correlaciones entre factores

\begin{tabular}{llllllll}
\hline & RRII & BMA & DEP & BEF & INC & DER & AUT \\
\hline RRII & & & & & & & \\
BMA & .324 & & & & & & \\
DEP & .430 & .277 & & & & \\
BEF & .591 & .405 & .582 & & & \\
INC & .325 & .225 & .371 & .355 & & \\
DER & .204 & .307 & .224 & .591 & .262 & & \\
AUT & .216 & .265 & .210 & .180 & .205 & .885 & \\
\hline
\end{tabular}

Nota: Siglas AUT: Autodeterminación, BEF: Bienestar emocional y físico; INC: Inclusión/ Presencia en la comunidad; RRII: Relaciones Interpersonales, BMA: Bienestar Material, DEP: Desarrollo Personal, DER: Derechos. 
Estos datos demuestran que este modelo es, comparativamente, el que mejor se ajusta a los datos. Con el fin de ofrecer una interpretación completa de los resultados, se analiza la fiabilidad compuesta y la varianza media extractada para cada uno de los constructos latentes del modelo 4 (Tabla 5).

La Tabla 5 muestra que las fiabilidades compuestas son elevadas (.637>IFC<.748). La varianza media extractada es superior al 50\% en todos los casos, excepto en relaciones interpersonales $(45.8 \%)$.

Tabla 5

Fiabilidad compuesta y varianza media extractada

\begin{tabular}{lcc}
\hline & IFC & AVE \\
\hline Relaciones interpersonales & .637 & .458 \\
Bienestar Material & .661 & .687 \\
Desarrollo personal & .666 & .563 \\
Bienestar emocional y físico & .748 & .547 \\
Inclusión & .666 & .774 \\
Derechos & .662 & .708 \\
Autodeterminación & .665 & .785 \\
Modelo & .936 & .640 \\
\hline
\end{tabular}

Nota. IFC: Índice de Fiabilidad Compuesta, AVE: Varianza Media Extractada.

Los valores de autodeterminación, inclusión y derechos informan positivamente de la validez y la fiabilidad de los indicadores empleados para explicar empíricamente los constructos latentes. Las dimensiones peor reflejadas en el modelo son desarrollo personal, bienestar emocional y físico y especialmente, relaciones interpersonales. En cuanto a la fiabilidad y validez del modelo, se han obtenido resultados muy satisfactorios (IFC $=.936 ; \mathrm{AVE}=64 \%$ ).

\section{Discusión y conclusiones}

Los dos primeros objetivos del estudio fueron el análisis de las propiedades psicométricas del CCVA y la validación del Modelo de Calidad de Vida de la Adolescencia que sustenta su creación. Los resultados de aplicar la escala a 2200 estudiantes indican un adecuado poder discriminativo de los ítems y una buena fiabilidad de la escala y de sus dimensiones.

Tras efectuar la parcelación de los ítems del instrumento, a fin de definir cada una de las dimensiones que componen al constructo calidad de vida, se llevó a cabo un AFC con el que se comprobó el ajuste del modelo original de Gómez-Vela \& Verdugo (2004, 2006), observándose un pobre ajuste de los datos. Por ello, fue reespecificado teniendo en cuenta la literatura de referencia. Los resultados mostraron que el modelo con siete componentes (autodeterminación, relaciones interpersonales, inclusión, desarrollo 
personal, derechos, bienestar emocional y físico, y bienestar material) fue el que mejor ajuste presentaba, con unos parámetros significativos en la mayoría de los indicadores.

Respecto al tercer objetivo, que pretendía analizar las relaciones entre las dimensiones de calidad de vida, se observan correlaciones positivas y adecuadas $(.21>\mathrm{r}<.90)$. En general, las correlaciones obtenidas aportan mayores evidencias de la validez de la escala al corresponderse con los resultados de las investigaciones previas.

Destaca, en primer lugar, la fuerte correlación existente entre las dimensiones autodeterminación y derechos, resultado que concuerda con investigaciones previas en las que se destaca la interconexión entre estos conceptos (López, Marín, \& de la Parte, 2004; Schalock\& Verdugo, 2002).

En segundo lugar, la elevada correlación de la dimensión relaciones interpersonales con bienestar emocional y físico e inclusión indica la interdependencia entre la disposición de relaciones valiosas con la familia, amigos y compañeros y de una red de apoyo social, integración y normalización, así como de sensación de satisfacción, felicidad y salud. En este sentido, el estudio de Chavarria \& Barra (2014) pone en valor la importancia del apoyo social percibido por el adolescente por parte de su familia, compañeros, profesorado y conocidos como factor de protección de su bienestar psicológico a lo largo de su vida.

Finalmente, la baja correlación entre autodeterminación y las demás dimensiones podría explicarse por la relativa independencia de la primera. Este resultado contrasta con las investigaciones de Arellano \& Peralta (2013), Biggs \& Carter (2016) y Wehmeyer \& Schwartz (1998) que evidencian que un nivel superior de autodeterminación favorece una mayor calidad de vida y una vida escolar y una adultez más positiva y satisfactoria.

En conclusión, aun sabiendo la presencia de limitaciones tales como el uso de una muestra que no es nacional ni aleatoria, es posible referirse a una serie de implicaciones teóricas y prácticas de este estudio: en primer lugar, que la calidad de vida evaluada en la población adolescente gallega mantiene la característica de multidimensionalidad propia de los modelos propuestos en las últimas décadas para su operativización; en segundo lugar, que este constructo, evaluado por medio del CCVA, presenta un comportamiento diferente en la población de esta Comunidad Autónoma en contraste con la propuesta original de Gómez-Vela y Verdugo (2004, 2006).

De tal forma, parece confirmarse la validez de un nuevo modelo aplicable a la población gallega, que no es otro que aquel que toma en consideración dimensiones relevantes del Modelo de Calidad de Vida en la Adolescencia y del Modelo Heurístico de Schalock \& Verdugo (2002). Bajo este marco conceptual, el uso de una herramienta útil y fiable como es el CCVA, podría ayudar a los centros educativos a obtener un diagnóstico de las necesidades, intereses, motivaciones y preocupaciones del alumnado, de modo que sea posible intervenir por medio de la promoción de sus dimensiones.

En definitiva, la evaluación de la calidad de vida se erige como eje clave para analizar las necesidades y demandas de la juventud, detectar las áreas de mayor y menor importancia en sus vidas, y planificar las intervenciones desde un enfoque centrado en la satisfacción individual. No obstante, teniendo en cuenta que los datos revelan que las diferencias entre regiones, patrones culturales y formas de vida local son un elemento diferenciador del bienestar de la adolescencia, se hace evidente que su medición exige atender a las características propias del grupo sujeto de evaluación. 


\section{Referencias}

Alfaro, J., Casas, F., \& López, V. (2015). Bienestar en la infancia y adolescencia. Psicoperspectivas, 14(1), 1-5. Recuperado de http://www.scielo.cl/scielo.php?script=sci_ arttext\&pid=S0718-69242015000100001

Arellano, A., \& Peralta, F. (2013). Calidad de vida y autodeterminación en personas con discapacidad. Valoraciones de los padres. Revista Iberoamericana de Educación, 63, 145-160. Recuperado de http://riberdis.cedd.net/handle/11181/4368

Arellano, A., \& Peralta, F. (2015). El enfoque centrado en la familia, en el campo de la discapacidad intelectual ¿cómo perciben los padres su relación con los profesionales?. Revista de Investigación Educativa, 33(1), 119-132. doi: 10.6018/rie.33.1.198561

Arias, B. (2008). Desarrollo de un ejemplo de análisis factorial confirmatorio con LISREL. En M.A. Verdugo, M. Crespo, M. Badía, \& B. Arias (Coords.), Metodología en la investigación sobre discapacidad. Introducción al uso de las ecuaciones estructurales (pp. 75-120). Salamanca: INICO.

Balboni, G., Coscarelli, A., Giunti, G., \& Schalock, R.L. (2013). The assessment of the quality of life of adults with intellectual disability: the use of self-report and report of others assessment strategies. Research in Developmental Disabilities, 34(11), 4248-4254. doi: 10.1016/j.ridd.2013.09.009.

Bandaloos, D.L., \& Finney, S.J. (2009). Item parceling issues in structural equation modeling. En G.A. Marcoulides, \& R.E. Schumaker (Eds.), New developments and techniques in structural equation modeling (pp. 269-296; 2a ed.). Mahwah, EE.UU.: Lawrence Erlbaum Associates.

Barbero, I. (Coord.), Vila, E., \& Holgado, F.P. (2010). Psicometría. Madrid: Sanz y Torres. Beadle-Brown, J., Leight, J., Whelton, B., Richardson, L., Beecham, J., Baumker, T., \& Bradshaw, J. (2015). Quality of life and quality of support for people with severe intellectual disability and complex needs. Journal of Applied Research in Intellectual Disabilities, 1, 1-13. doi: 10.1111/jar.12200

Biggs, E.E., \& Carter, E.W. (2016). Quality of life for transition-age youth with autism or intellectual disability. Journal of Autism and Developmental Disorders, 46(1), 190204. doi: 10.1007/s10803-015-2563-x.

Chavarria, M.P., \& Barra, E. (2014). Satisfacción vital en adolescentes: relación con la autoeficacia y el apoyo social percibido. Terapia Psicológica, 32(1), 41-46. doi: $10.4067 /$ S0728-4808201400010000

Claes, C., van Hove, G., van Loon, J., Vandevelde, S., \& Schalock, R.L. (2010). Quality of life measurement in the field of intellectual disabilities: Eight principles for assessing quality of life-related personal outcomes. Social Indicators Research, 98(1), 61-72. doi: 10.1007/s11205-009-9517-7

Escarabajal, A., Martínez, S., \& Salmerón, J.A. (2015). La percepción de la calidad de vida de las mujeres mayores y su envejecimiento activo a través de actividades socioeducativas en los centros sociales. Revista de Investigación Educativa, 33(2), 471-488. doi: 10.6018/rie.33.2.213211

Escobedo Portillo, M.T., Hernández Gómez, J.A., Estebané Ortega, V., \& Martínez Moreno, G. (2016). Modelos de ecuaciones estructurales: Características, fases, construcción, aplicación y resultados. Cienc Trab, 18(55), 16-22. doi: 10.4067/S071824492016000100004 
Gómez-Sánchez, L.E., Peña, E., Arias, B., \& Verdugo, M.A. (2016). Impact of individual and organizational variables on quality of life. Social Indicators Research, 125, 649664. doi: 10.1007/s11205-014-0857-6

Gómez-Vela, M. (2007). La calidad de vida de alumnos con necesidades educativas especiales y sin ellas: Elaboración de un marco conceptual y un instrumento de evaluación. Educación y Diversidad, 1, 113-136. Recuperado de https://dialnet. unirioja.es/servlet/articulo?codigo $=2313563$

Gómez-Vela, M., \& Verdugo, M.A. (2004). El cuestionario de evaluación de la calidad de vida de alumnos de educación secundaria obligatoria: Descripción, validación inicial y resultados obtenidos tras su aplicación en una muestra de adolescentes con discapacidad y sin ella. Siglo Cero: Revista Española sobre Discapacidad Intelectual, 35(212), 5-17. Recuperado de http://riberdis.cedd.net/handle/11181/3096

Gómez-Vela, M., \& Verdugo, M.A. (2006). La calidad de vida en la adolescencia: evaluación de jóvenes con discapacidad y sin ella. En M.A. Verdugo (Ed.), Cómo mejorar la calidad de vida de las personas con discapacidad: Instrumentos y estrategias de evaluación (pp. 77-102). Salamanca: Amarú.

Gómez-Vela, M., \& Verdugo, M.A. (2009). (Eds). Cuestionario de evaluación de la calidad de vida en alumnos adolescentes (CCVA). Madrid: CEPE.

Gómez-Vela, M., Verdugo, M.A., \& González-Gil, F. (2007). Calidad de vida y autoconcepto en adolescentes con necesidades educativas especiales y sin ellas. Infancia y Aprendizaje, 30(4), 523-536. doi: 10.1174/02103700778334300

Higuita, L.F., \& Cardona, J.A. (2015). Concepto de calidad de vida en la adolescencia: Una revisión crítica de la literatura. Revista CES Psicología, 8(1), 155-168. Recuperado de http://revistas.ces.edu.co/index.php/psicologia/article/view/3031

Instituto Galego de Estatística (2015). Ensinanza non universitaria. Alumnado matriculado en centros segundo sexo, nivel de ensinanza e titularidade do centro [Fuente de Datos y Libro de Códigos]. Recuperado de https://www.ige.eu/igebdt/selector.jsp?COD=22 90\&paxina $=001 \& c=0203002$

International Association for the Scientific Study of Intellectual Disabilities. Special Interest Research Group on Quality of Life (2000). Quality of life: its conceptualization, measurement, and application. A consensus document. Ginebra, Suiza: World Health Organization.

Kelloway, E.K. (2014). Using Mplus for structural equation modeling: A researcher's guide (2 $2^{\mathrm{a}}$ ed.). Nueva York, EE.UU.: Sage.

Kenny, D.A., \& Milan, S. (2014). Identification: a nontechnical discussion of a technical issue. En R.H. Hoyle (Ed.), Handbook of structural equation modeling (pp. 145-163). Nueva York, EE.UU.: Guilford Publications Inc.

Kline, R.B. (2015). Principles and practice of structural equation modeling ( $4^{\mathrm{a}}$ ed.). Nueva York, EE.UU.: The Guildford Press.

Lang, K.M., \& Little, T.D. (2016). Principled missing data treatment. Prevention Science, 4, 1-11. doi: 10.1007/s11121-016-0644-5

López, M.A., Marín, A.I., \& de la Parte, J.M. (2004). La planificación centrada en la persona, una metodología coherente con el respeto al derecho de autodeterminación. Siglo Cero: Revista Española Sobre Discapacidad Intelectual, 35(1), 45-55. Recuperado de http://riberdis.cedd.net/handle/11181/3093 
Malone, P.S., \& Lubansky, J.B. (2014). Preparing data for structural equation modeling: Doing your homework. En R.H. Hoyle (Ed.), Handbook of structural equation modeling (2 $2^{\mathrm{a}}$ ed., pp. 263-276). Nueva York, EE.UU.: The Guilford Press.

Mateo, J., \& Martínez, F. (2008). Medición y evaluación educativa. Madrid: La Muralla.

Morisse, F., Vandemaele, E., Claes, C., Claes, L., \& Vandevelde, S. (2013). Quality of life in persons with intellectual disabilities and mental health problems: an explorative study. The Scientific World Journal, 1, 1-8. doi.: 10.1155/2013/491918

Muntaner, J.J. (2013). Calidad de vida en la escuela inclusiva. Revista Iberoamericana de Educación, 63, 35-49. Recuperado de https://dialnet.unirioja.es/servlet/ articulo? codigo $=4773546$

Schalock, R.L., \& Verdugo, M.A. (2002). (Eds.). Quality of life for human service practitioners. Washington, EE.UU.: AAMR.

Schalock, R.L., Verdugo, M.A., Gómez, L.E., \& Reinders, H.S. (2016). Moving us toward a theory of individual quality of life. American Journal on Intellectual and Developmental Disabilities, 121(1), 1-12. doi: 10.1352/1944-7558-121.1.1

Vega, V., Jenaro, C., Flores, N., Cruz, M., \& Lerdo, A. (2013). Evaluación de la calidad de vida de adultos con discapacidad intelectual institucionalizados en Chile. Universitas Psychologica, 12(2), 471-481. doi.: 10.11144/Javeriana.UPSY12-2.ecva

Verdugo, M.A., Sainz, F., Gómez-Sánchez, L.E., \& Gómez-Santamaría, S. (2011). Bases para el desarrollo de un modelo evaluación para personas con discapacidad intelectual adultas que viven en servicios residenciales [versión Adobe Digital Editions]. Retrieved from http://sid.usal.es/25938/8-1

Wehmeyer, M. \& Schwartz, M. (1998).The relationship between self-determination and quality of life for adults with mental retardation. Education and Training in Mental Retardation and Developmental Disabilities, 33(1), 3-12. Recuperado de https://www. jstor.org/stable/23879037?seq=1\#page_scan_tab_contents

West, S.G., Taylor, A.B., \& Wu, W. (2015). Model fit and model selection in structural equation modeling. En R.H. Hoyle (Ed.), Handbook of structural equation modeling ( $2^{\mathrm{a}}$ ed., pp. 209-231). Nueva York, EE.UU.: The Guilford Press.

Fecha de recepción: 1 de febrero de 2018 Fecha de revisión: 28 de febrero de 2018 Fecha de aceptación: 23 de abril de 2018 
